\title{
Madame Tussaud et le masque de Robespierre. Exercices d'histoire autour de la médiatique reconstitution d'un visage
}

Hervé Leuwers et Guillaume Mazeau

\section{(2) OpenEdition \\ Journals \\ Édition électronique \\ URL : https://journals.openedition.org/ahrf/13083 \\ DOI : 10.4000/ahrf.13083 \\ ISSN : 1952-403X \\ Éditeur : \\ Armand Colin, Société des études robespierristes}

Édition imprimée

Date de publication : 1 mars 2014

Pagination : 187-198

ISBN : 978-2-200-9083-2790-8

ISSN : 0003-4436

Référence électronique

Hervé Leuwers et Guillaume Mazeau, « Madame Tussaud et le masque de Robespierre. Exercices d'histoire autour de la médiatique reconstitution d'un visage », Annales historiques de la Révolution française [En ligne], 375 | janvier-mars 2014, mis en ligne le 01 juillet 2017, consulté le 05 juillet 2021. URL : http://journals.openedition.org/ahrf/13083 ; DOI : https://doi.org/10.4000/ahrf.13083 


\section{MADAME TUSSAUD ET LE \\ MASQUE DE ROBESPIERRE \\ EXERCICES D'HISTOIRE AUTOUR DE LA \\ MÉDIATIQUE RECONSTITUTION D'UN VISAGE}

Hervé LEUWERS et Guillaume MAZEAU

En 1911, par un remarquable article publié dans les Annales révolutionnaires, puis largement diffusé sous forme de brochure, Hector Fleischmann croyait en finir avec la légende des masques mortuaires de Robespierre ${ }^{1}$. Mais les mythes ont la vie dure ; par une médiatique reconstitution du visage du conventionnel, doublée d'un diagnostic rétrospectif de ses pathologies, le docteur Philippe Charlier et Philippe Froesch, de l'entreprise Visualforensic, viennent de le démontrer...

Rappelons d'abord la cocasse affaire de mars 1911. Elle se déroule à Paris, lorsque le musée Carnavalet ouvre de nouvelles salles au public, consacrées à la Révolution et à l'Empire. Parmi les meubles issus de la prison du Temple, les bustes, les tableaux et les médailles, se détache un saisissant masque de cire de Robespierre. Le visage est farouche, mais émacié, avec les yeux clos et les lèvres serrées ; une blessure s'aperçoit au bas de la mâchoire. L'objet, précise le catalogue, « semblait provenir du fameux musée d'images de cire que Curtius avait fondé sur le boulevard du Temple ». Relayant une explication avancée lors de la précédente exposition du masque (1882), le journal Le Temps rapporte, de son côté, qu'une autre interprétation l'attribue à Palloy, l'entrepreneur qui a détruit la Bastille et diffusé tant de souvenirs patriotiques ; il l'aurait réalisé « sur nature », au pied de l'échafaud.

(1) Hector FLEISCHMANN, « Le masque mortuaire de Robespierre », Annales révolutionnaires, 1911, p. 601-625 ; l'article est disponible sous la forme d'une brochure : Le masque mortuaire de Robespierre. Documents nouveaux pour servir d'intelligence et de conclusion à une polémique historique, Paris, Leroux, 1911. 
L'exposition à peine ouverte, un article du Matin (d'Anvers) lance la polémique ${ }^{2}$. Camille Liaume y assure que le masque est un faux, qui lui a un moment appartenu et a été réalisé par le sculpteur Maurice Castan. Avec précision, il éclaire son cheminement, de sa première exposition lilloise (1882) à son don à Carnavalet, par la veuve de Charles Simon. Pendant quelques jours, les gazettes s'emballent et s'amusent. Prudent, le directeur de Carnavalet en accepte le verdict et retire le masque de l'exposition « jusqu'à plus ample informé ». Depuis, il demeure dans les réserves du musée $^{3}$; il est vrai qu'à elle seule, la position de la blessure, à droite du visage (et non à gauche !), a confirmé - tardivement - la supercherie. Mais d'autres masques existent...

Ainsi, un peu plus d'un siècle plus tard, l'un d'entre eux refait surface et inspire une modélisation du visage de Robespierre, présentée comme une "découverte » à caractère scientifique. Appuyée par une intense campagne de communication, elle reçoit la caution d'une partie de la presse et du monde médical. Le 11 octobre 2013, lors de la Journée de la Science ${ }^{4}$, Philippe Froesch présente le résultat de son travail au muséum d'histoire naturelle d'Aix-en-Provence, qui a fourni la copie du masque mortuaire. Deux mois plus tard, après avoir annoncé une prochaine « révélation », Philippe Froesch et le médecin Philippe Charlier, lui-même habitué du monde des médias ${ }^{5}$, présentent l'image numérisée du visage de Robespierre. Une nouvelle fois, la date de la conférence de presse a été choisie avec soin : le lendemain, la célèbre revue médicale The Lancet met en ligne une lettre décrivant la maladie jusqu'ici inconnue de Robespierre : la sarcoïdose qui, selon Philippe Charlier, expliquerait l'épuisement (supposé) du révolutionnaire ${ }^{6}$. Dans les jours précédents, une marée d'articles paraphrasant le même dossier de presse a déjà loué la 《 prouesse scientifique et technique $»^{7}$ permettant enfin de « révéler » ou « dévoiler » la « vraie nature » de l'Incorruptible. La bataille du public a paru alors gagnée. Sur internet, la nouvelle inspire en effet des milliers de

(2) Ibid. ; voir aussi : «Le Robespierre en cire du musée Carnavalet », Annales révolutionnaires, 1911, p. 425-428 ; « Le masque de Robespierre », Revue historique de la Révolution française, 1911, p. 315-316.

(3) Musée Carnavalet, S-2075. Nous tenons à remercier Philippe de Carbonnières et Philippe Sorel, pour les informations qu'ils nous ont communiquées sur les collections du musée Carnavalet.

(4) museum-aix-en-provence.org/Maximilien_Robespierre_3D.pdf.

(5) Intervenant régulier dans des émissions comme Secrets d'Histoire, il présente depuis novembre 2013 une série de documentaires sur Arte intitulée « Enquête d'ailleurs ».

(6) Philippe CHARLIER, Philippe FROESCH, « Robespierre : the oldest case of sarcoidosis ?», The Lancet, Volume 382, Issue 9910, Page 2068, 21 December 2013.

(7) LCI, 14 décembre 2013, journal de 12 h : http://videos.tf1.fr/infos/2013/le-visage-derobespierre-reconstitue-en-3d-8330323.html. 
commentaires. Au début du mois de janvier 2014, l'article de L'Alsace.fr qui a été le premier à mettre en ligne le visage de Robespierre atteint presque 10000 lectures $^{8} . .$.

Très tôt, pourtant, plusieurs voix dissonantes se sont élevées, d'abord dans le monde politique et en particulier dans une partie de l'extrême gauche, attachée à la mémoire positive de l'Incorruptible. Le 13 décembre, le secrétaire national du Parti de gauche, Alexis Corbière, bientôt suivi par Jean-Luc Mélenchon, perçoit l'image présentée au public comme monstrueuse, et dénonce une entreprise de destruction de la Révolution tout entière ${ }^{9}$. De telles réactions montrent à quel point l'événement joue comme un révélateur : encore en 2013, la mémoire de Robespierre et des années 1793-1794 demeure une question sensible. De leur côté, les historiens universitaires ne sont pas restés muets. Annie Duprat relaie la nouvelle sur twitter de manière distanciée dès le 13 décembre, confirmant ses doutes quatre jours plus tard ${ }^{10}$. Le soupçon est partagé par Emmanuel Laurentin, producteur de l'émission de radio La Fabrique de l'Histoire, mais aussi par Marc Belissa et Yannick Bosc, récents auteurs d'un livre sur Robespierre et animateurs du blog révolution-française.net, ou par Philippe de Carbonnières, du musée Carnavalet ${ }^{11}$. Entre temps, nous sommes intervenus dans le débat. Le 17 décembre, Guillaume Mazeau publie un billet sur le site du Comité de Vigilance sur les Usages Publics de l'Histoire, repris par une partie de la presse et de la blogosphère ${ }^{12}$. Trois jours plus tard, Bernadette Arnaud relaie les doutes d'Hervé Leuwers dans Sciences et Avenir, alors que, le soir, France 2 et France 3 Nord-Pasde-Calais, consacrent un sujet à ce qui devient l' «affaire Robespierre », présentée par des dizaines de journaux dans le monde anglo-saxon comme une $«$ controverse majeure $»^{13}$. Imanol Corcostequi, du site rue89, fait quant à lui la part belle aux critiques historiennes et au débat, ouvrant un

(8) http://www.lalsace.fr/actualite/2013/12/12/on-a-rendu-sa-tete-a-robespierre.

(9) http://www.alexis-corbiere.com/index.php/post/2013/12/15/Faux-masque-en-3D-etauthentiques-calomnies-antirobespierristes. Voir aussi la chronique de Pierre Marcelle, «Zéro + zéro = la tête à Maximilien Robespierre », Libération, 19 décembre 2013.http://www.jean- luc-melenchon. fr/2013/12/16/de-bogota/\# article1.

(10) https://twitter.com/DupratA/status/412999013185499136.

(11) http://revolution-francaise.net/2013/12/13/555-la-tete-kitsch-de-robespierre. Marc BELISSA, Yannick Bosc, Robespierre. La fabrication d'un mythe, Paris, Ellipses, 2012. http://www. huffingtonpost.com/2013/12/20/robespierre-death-mask-makeover-diagnosis-photo_n_4481018. html.

(12) http://cvuh.blogspot.fr/2013/12/le-vrai-visage-de-robespierre-par.html.

(13) France $2:$ http://www.francetvinfo.fr/replay-jt/france-2/20-heures/jt-de-20h-duvendredi-20- decembre-2013_482330.html. France 3: http://www.francetvinfo.fr/sciences/videola- reconstitution-du-visage-de-robespierre-cree-la-polemique_489246.html. Voir aussi l'article sur huffingtonpost.com, cité note 11 . 
droit de réponse à Philippe Froesch ${ }^{14}$. Cette fois, les historiens ont joué un vrai rôle dans l'espace public et tout particulièrement sur internet.

Pris dans un temps très court, beaucoup de médias n'ont pas saisi les enjeux plus profonds de ce qu'ils ont réduit à une simple «polémique ». Et pourtant, le visage large et imposant, la peau rougie et trouée de crevasses, les petits yeux perçants de ce Robespierre en 3D ne sont évidemment pas neutres. Volontairement ou non, les auteurs de la reconstitution s'inscrivent dans une tradition satirique et tératologique, faisant de Robespierre un des « monstres » de la Révolution ; l'épaisseur, les plis et les marques du visage trahissent leur perception des acteurs d'une révolution passée, qui n'est pas sans trahir les frayeurs et angoisses de nos sociétés occidentales. Le thème de la maladie renvoie quant à lui à une interprétation pessimiste de l'histoire, assimilant la Révolution à une dégénérescence organique, qui n'épargne pas ses principaux acteurs. Si les historiens ont pris la peine d'intervenir, ce n'est pas d'autorité, mais parce qu'ils sont en mesure, grâce à leur expérience, d'atténuer la sidération de leurs concitoyens devant les «nouvelles technologies » à la lumière d'une histoire, bien plus longue, du mythe de Robespierre.

S'il est strictement impossible d'intenter un procès d'intention à Philippe Froesch et Philippe Charlier, il faut convenir que la nature même de leur projet pèse lourdement sur la fiabilité de leur produit. Visualforensic est une société privée domiciliée à Barcelone, spécialisée dans les reconstructions faciales en images de synthèse, les impressions 3D en résine et les identifications faciales. Contrainte de trouver des marchés mondiaux, elle concentre son activité de communication autour de grands personnages de l'histoire ou de figures anonymes emblématiques, susceptibles d'attirer une audience internationale (momie égyptienne, Henri IV, Bolivar, évidemment populaire dans le monde ibérique) et/ou communautaire («victime d'un pogrom du XIV $\mathrm{XV}^{\mathrm{e}}$ siècle $\left.»\right)^{15}$. L'entreprise est liée au Groupe de recherche en pathologie (GROB) de l'Université Autonome ainsi qu'à la Faculté de médecine de Barcelone. Affirmant utiliser des procédés d'identification faciale de la police scientifique (Philippe Froesch a ainsi précisé avoir reconstitué

(14) Imanol Corcostequi, « Pourquoi la reconstitution du visage de Robespierre énerve les historiens », rue89, 24 décembre 2013 : http://www.rue89.com/2013/12/24/pourquoi-reconstitutionvisage-robespierre-enerve-les-historiens-248566; La réponse de Philippe Froesch : «Auteurs de la reconstitution du visage de Robespierre, nous n'avons pas voulu le dénigrer », rue89, 27 décembre 2013 : http://www.rue89.com/2013/12/27/auteurs-reconstitution-visage-robespierre-navonsvoulu-denigrer-248629.

(15) http://www.visualforensic.com/. 
le visage de Robespierre à partir de techniques utilisées par le FBI ${ }^{16}$, l'entreprise propose également des expertises dans le cadre d'enquêtes judiciaires. Visualforensic apparait ainsi d'un statut hybride, et navigue entre le monde de l'entreprise et de la recherche scientifique publique, voire le monde de l'art, Philippe Froesch se présentant lui-même comme un « Forensic artist » - en l'occurrence, un infographiste médico-légal. Surtout, depuis plusieurs années, l'entreprise travaille avec le médecin légiste, anathomo-pathologiste et paléopathologiste Philippe Charlier, maître de conférences et praticien hospitalier dans le service de médecine légale de l'Hôpital universitaire Raymond Poincaré de Garches (AP-HP, Université de Versailles/Saint-Quentin-en-Yvelines). Celui-ci s'est lui aussi spécialisé dans l'analyse des restes réels ou supposés de figures illustres de l'histoire comme Richard Cœur de Lion, Agnès Sorel, Diane de Poitiers, Jeanne d'Arc ou Henri IV, dont la tête présumée, déjà modélisée par Philippe Froesch, a provoqué les doutes de plusieurs historiens ${ }^{17}$.

Dans cette affaire, la technique n'est pas en cause, tant les procédés numériques de visualisation ont démontré leur intérêt heuristique. La sociologie, les sciences politiques, la géographie et l'histoire s'enrichissent de l'utilisation de nouveaux outils numériques permettant de modéliser des données statistiques, d'analyser des textes, de cartographier ou de reconstituer des espaces inaccessibles, immatériels ou disparus, de diffuser les outils et les résultats de la recherche ou, plus profondément encore, de communiquer et de travailler de manière différente. À condition qu'elle s'appuie sur des sources fiables, la reconstitution de visages du passé n'est en particulier pas dépourvue d'intérêt, comme le montre l'actuel développement des Visual Studies: savoir à quoi ont pu ressembler Robespierre, Olympe de Gouges ou Bailly, pour lesquels les historiens ne disposent que de descriptions littéraires ou iconographiques souvent trompeuses, peut, en révélant les décalages entre leur réalité physique et de telles images, aider à approcher les questions des représentations, du charisme, de l'individualité, des représentations collectives, du beau et du laid, des stigmates de la maladie sur les corps et dans les esprits, du genre ou de l'identité sociale. De la même manière, malgré les nombreuses fragilités de la paléopathologie, malgré la difficulté de poser des diagnostics concernant des individus décédés depuis plusieurs siècles, en l'absence de restes organiques, savoir de quel mal ont vraiment souffert Marat, Mirabeau

(16) http://nord-pas-de-calais.france3.fr/2013/12/14/des-chercheurs-viennent-dereconstituer-le-visage-de-maximilien-robespierre-377483.html.

(17) Joël Cornette, «L'étrange affaire du crâne d'Henri IV », sur histoire.presse.fr. 
ou Robespierre est une question qui mérite d'être posée, et peut approfondir les connaissances en matière d'histoire démographique, médicale, sociale, mais aussi politique et culturelle : à la fin du XVIII ${ }^{\mathrm{e}}$ siècle, la santé des « hommes politiques » fait l'objet d'intenses références, Robespierre luimême n'hésitant pas à se dire prêt au sacrifice pour le peuple et la patrie. Le problème n'est donc ni la discipline ni la technique, mais la méthode, et avant toute chose le choix de la source : la copie du masque mortuaire de Robespierre, conservée au muséum d'Aix-en-Provence, censée avoir été pratiquée sur un original réalisé in vivo, si l'on peut dire, par la future Mme Tussaud.

À la question, "possédons-nous un moulage du visage de Robespierre? », Hector Fleischmann répond sans hésitation par la négative $^{18}$; un siècle plus tard, l'affirmation reste souvent aussi ferme ${ }^{19}$. Il ne s'agit pas, bien sûr, de nier l'existence de figures de cire représentant Robespierre. L'une d'elles a existé dans le musée Curtius où, d'après le témoignage de Delécluze, Jacques-Louis David l'aurait vue sous l'Empire, la mâchoire retenue par un bandeau ; le peintre l'aurait jugée bien imitée et bien faite ${ }^{20}$. L'une de ces têtes de cire se trouve dans les collections du musée Tussaud ; des photographies anciennes la montrent ceinte d'un bandage. À Paris, de tels objets ont fait le succès de Curtius, dès avant la Révolution $^{21}$; c'est dans son musée que Marie Grosholtz, nièce et pupille de Curtius, et future Mme Tussaud, a fait son apprentissage. Les figures exposées, précisément reproduites, font alors fréquemment référence à l'actualité ; une galerie de portraits du début du XIX ${ }^{\mathrm{e}}$ siècle laisse imaginer les bustes de contemporains tantôt disparus, comme Charlotte Corday, Marat ou le comte de Lorges (dont nous aurons à reparler), et tantôt vivants, comme Bonaparte ${ }^{22}$.

C'est à partir d'un masque mortuaire en plâtre, et non de la figure de cire du musée Tussaud, que Philippe Froesch a reconstitué le prétendu visage de Robespierre. La comparaison avec les documents du Musée Tussaud, précise-t-il, sera faite dans les mois prochains ; soit. D'emblée, pourtant, il a affirmé que le masque d'Aix-en-Provence n'est qu'une copie

(18) Hector FLEISCHMANN, op. cit., p. 601, 620.

(19) Antoine de BAECQUE, «Le tableau d'un cadavre. Les récits d'agonie de Robespierre : du cadavre hideux au dernier héros », dans Annie JOURDAN (dir.), Robespierre - Figure réputation, Amsterdam, Rodopi, 1996, p. 176.

(20) Cité dans Hector Fleischmann, op. cit., p. 614-616.

(21) Jean ADHÉMAR, «Les musées de cire en France, Curtius, le 'banquet royal', les têtes coupées », Gazette des beaux-arts, décembre 1978, p. 203-214.

(22) Biographical sketches of the Characters Composing the Cabinet of Composition Figures Executed by The Celebrated Curtius of Paris and his Successor, Edinburgh, 1803. 
de celui de Londres - ce qui mériterait vérification. Que sait-on ? Le plâtre du muséum d'histoire naturelle d'Aix-en-Provence paraît identique au moulage de la collection Dumoutier (Muséum national d'histoire naturelle), mais aussi à celui de la collection Hutton (Université de Princeton). Ajoutons qu'il est également au catalogue du mouleur d'art Lorenzi, en activité depuis 1871, qui le reproduit sur demande en plâtre, résine, pierre, bronze ou terre cuite ${ }^{23} . .$. Lors de l'exposition que le musée d'Orsay a consacrée au Dernier portrait, en 2002, c'est d'ailleurs un masque de plâtre provenant de ce célèbre atelier qui a été exposée ${ }^{24}$.

Incontestablement, le masque est d'origine ancienne et existe à de nombreux exemplaires; par plusieurs photographies de la fin du XIX siècle et du début du $\mathrm{XX}^{\mathrm{e}}$, on peut en identifier une copie dans la collection de M. Leclercq, de Compiègne, dont le moulage porte une étiquette datée de 1832, qui précise : «Cette épreuve a été tirée sur l'empreinte originale qui fut moulée sur la figure de Robespierre, immédiatement après qu'il eut été guillotiné, et qui appartient au citoyen Turbri, compositeur de musique ». $\mathrm{Au}$ début du $\mathrm{XX}^{\mathrm{e}}$ siècle, nous apprend Hector Fleischmann, la même origine est mentionnée sur les masques détenus par Gabriel Thomas et par Léon Moreaux, l'exemplaire de ce dernier ayant été exposé à Paris en $1889^{25}$. François Laurent Hébert Turbri (1795-1859) a-t-il acquis son moulage en 1826, à l'occasion de la vente des collections de Dominique-Vivant Denon, qui comprend plusieurs prétendus masques funéraires, dont celui de Robespierre ? Plusieurs indices, dont Hector Fleishmann n'a pu disposer, semblent plaider pour cette hypothèse : la taille de l'objet est identique ${ }^{26}$, et une lithographie du début XIX ${ }^{\mathrm{e}}$ siècle, présentant deux études du masque, pourrait être de Denon. Le catalogue de la vente de 1826, qui mentionne

(23) Catalogue de la Maison Lorenzi, référence 947 (précisons que, pour se procurer le masque, il en coûte $112 €$ en plâtre brut, et $147 €$ en plâtre patiné blanc - prix au 9 janvier 2014). $33,213$.

(24) Le dernier portrait. Musée d'Orsay. Paris, 5 mars - 26 mai 2002, Paris, RMN, 2002, p.

(25) Hector FleISCHMANN, op. cit., p. 622-623 ; Docteur CABANÈS, Les indiscrétions de l'histoire, 5e série, Paris, Albin Michel, 1952, p. 357 ; «Le Robespierre en cire...», op. cit., p. 428 ; Catalogue des objets formant l'exposition historique de la Révolution française. Salle des États, aux Tuileries, place du Carrousel, Paris, au siège de la société de l'histoire de la Révolution française, 1889 , p. 12. Précisons que le masque détenu par le muséum national d'histoire naturelle porte l'étiquette Turbri. Les informations sur les masques conservés au muséum national d'histoire naturelle (MNHN-HA-D-36) et au muséum d'histoire naturelle d'Aix-en-Provence (1860-108-1), nous ont été aimablement fournies par Aurélie Fort, assistante de conservation au MNHN, et Gilles Cheylan, conservateur en chef du patrimoine au muséum d'Aix-en-Provence, que nous remercions.

(26) Le catalogue de la vente Denon précise qu'il mesure 9 pouces et demi de hauteur (soit $25,7 \mathrm{~cm}$ ), et la copie du masque Turbri, par la Maison Lorenzi, mesure $25 \mathrm{~cm}$. 
une «empreinte moulée sur le visage de Robespierre, avant sa mort », invite cependant à la prudence ${ }^{27}$.

Quoi qu'il en soit, remarquons que, du catalogue de la vente Denon (1826) à celui de l'exposition de 1889, et à l'inventaire iconographique de Buffenoir (1909), ce masque que nous appellerons Turbri, du nom de son plus ancien propriétaire connu avec certitude, n'est jamais attribué à Mme Tussaud $^{28}$. Il est vrai que ses mémoires, où elle assure avoir pris l'empreinte du visage du conventionnel exécuté, paraissent en 1838 seulement, et en anglais ; ce n'est qu'au début du $\mathrm{XX}^{\mathrm{e}}$ siècle, que le masque est ainsi directement relié à elle, notamment par Hector Fleischmann qui, sur ce point, ne cherche pas à étayer son affirmation. L'attribution se retrouve dans le catalogue de la collection de l'Université de Princeton, et dans celui de l'exposition du musée d'Orsay de 2002. Elle a été reprise par Philippe Froesch et Philippe Charlier. N'est-il pas étonnant, pourtant, que les auteurs du $\mathrm{XIX}^{\mathrm{e}}$ siècle ne mentionnent aucunement cette provenance ? Compte tenu de la célébrité de l'oncle Curtius, et bientôt de Mme Tussaud elle-même, elle aurait renforcé l'authenticité possible du moulage... Faute de pouvoir trancher sur ce point, poursuivons la réflexion en considérant que nos deux auteurs ont raison dans leur attribution.

Pour Philippe Froesch et Philippe Charlier, les choses sont simples ; Mme Tussaud affirme avoir réalisé le masque de Robespierre, et sa formation lui a permis de le faire, on peut donc la croire sur parole... Pourtant, quel étrange livre que ces Memoirs and Reminiscences de Mme Tussaud! Au mieux, il n'est qu'un réjouissant roman où, entre quelques souvenirs crédibles et des relations d'événements assez convenues, s'accumulent poncifs et anecdotes invraisemblables. Depuis 2005, l'ouvrage est traduit en français et accessible à tous ${ }^{29}$; inutile, donc, d'en faire une critique serrée. Contentons-nous de relever quelques amusantes invraisemblances en lien direct avec Robespierre.

En voici une première. Après le 14 juillet, rapportent les mémoires, alors qu'elle visite la Bastille avec son oncle et des amis, Marie Grosholtz aurait glissé dans les escaliers du château et aurait été rattrapée par... Robespierre ; «il fit alors galamment observer que c'eût été une grande pitié qu'une si jeune et jolie patriote se fût brisé le cou dans un endroit

(27) Dominique-Vivant DENON, L'œil de Napoléon, Paris, RMN, 1999, p. 394 (figure 121). Le dernier portrait, op. cit., p. 95.

(28) Hector FleISCHMANN, op. cit., p. 622 ; Catalogue des objets..., op. cit., 1889, p. 12 ; Hippolyte BUfFENOIR, Les portraits de Robespierre, Paris, Leroux, 1909, p. 27 («Après la mort de Robespierre, écrit-il, on prit un moulage de ses traits »; il cite ensuite le moulage exposé en 1889).

(29) Marie TuSSAUD, Mémoires et souvenirs sur la Révolution française, Paris, Arléa, 2005. 
aussi affreux ». Peu avant, elle écrit qu'on lui a présenté le plus ancien et célèbre prisonnier de la Bastille, le comte de Lorges, "afin qu'elle prit un moulage de sa tête qu'elle compléta ensuite et qu'elle possède encore dans sa collection. Parfaitement ressemblant, c'est la vie même ». Malheureusement pour la crédibilité de Mme Tussaud, le comte de Lorges n'a jamais existé ; c'est un mythe, bien connu des historiens ${ }^{30}$ ! Poursuivons tout de même. Influencée par certaines légendes thermidoriennes, qui ont attribué à Robespierre une maison de plaisir à Issy, Mme Tussaud brosse le portrait d'un « libidineux », avide de conquêtes et prêt à envoyer des innocents à la guillotine pour saisir leurs biens en faveur de ses maîtresses. Enfin, dernière information capitale : Mme Tussaud précise que son moulage a été réalisé au cimetière de la Madeleine ${ }^{31} \ldots$ Or Robespierre et les autres exécutés du 10 thermidor ont été ensevelis au cimetière des Errancis ! Aurait-elle pu l'oublier?

Tout cela, on en conviendra, fragilise le témoignage de Mme Tussaud. De plus, comment croire qu'elle ait pu approcher du corps supplicié, prendre sa tête tranchée et blessée, et en faire le masque ? Alors que le gouvernement souhaite faire disparaître les corps du 10 thermidor sous la chaux vive, alors qu'il craint la constitution d'un lieu de mémoire, alors qu'on ne plaisante pas avec ses ordres, comment imaginer que le bourreau aurait pu prendre le risque d'autoriser ce geste ? Ni elle, ni aucune autre personne, sans doute, n'a pu s'approcher de ce cadavre que l'on continue de craindre. Bien des aspects du masque Turbri, d'ailleurs, devraient étonner. Le témoignage des chirurgiens qui ont examiné Robespierre, quelques heures avant son exécution, précisent qu'ils ont «apperçu d'abord un gonflement à toute la face, plus considérable à gauche (le côté blessé) ; il y avoit aussi érosion à la peau et chymose à l'œil du même côté $»^{32}$. Et rien de tout cela n'aurait été perceptible sur le masque funéraire ? Aucun gonflement, aucune déformation, aucune marque évidente à l'emplacement de la blessure?

Et que penser de l'étrangeté du visage reconstitué, qui ne rappelle en rien les portraits connus du révolutionnaire ? Certes, l'ancienne étude iconographique de Buffenoir est à reprendre ${ }^{33}$; plusieurs des portraits qu'il mentionne ne sont plus reconnus comme étant de Robespierre, et

(30) Marie TUSSAUD, op. cit., p. 68. Voir Rolf REICHARDT, L'imagerie révolutionnaire de la Bastille. Collections du Musée Carnavalet, Paris, Paris-Musées, 2009, p. 108-111.

(31) Marie TuSSAUD, op. cit., p. 146-148.

(32) Edme Bonaventure COURTOIS, Rapport fait au nom des comités de Salut public et de Sûreté générale sur les événements du 9 thermidor an II..., Paris, imprimerie nationale, an IV, p. 202. (33) Hippolyte BufFENOIR, Les portraits de Robespierre, Paris, Leroux, 1909. 
de nouvelles identifications ont été à leur tour remises en cause. Ainsi, le tableau de Vigneron conservé aux châteaux de Versailles et de Trianon est encore souvent présenté comme une copie du pastel qu'Adélaïde LabilleGuiard a exposé au salon de septembre 1791 ; les spécialistes de l'artiste, pourtant, n'y reconnaissent aucunement sa patte, et doutent fortement de l'identité du modèle ${ }^{34}$. Le portrait de Boilly du musée de Lille, identifié à Robespierre par un article de 1928 dont les minces arguments mériteraient d'être sérieusement discutés, peut également susciter le doute ${ }^{35}$ !

Si l'on s'en tient à quelques représentations indiscutables, et particulièrement au portrait anonyme, conservé par le Musée Carnavalet, on peut souligner un caractère physique de Robespierre, totalement absent du masque funéraire. Sur ces représentations, en effet, comme sur nombre de gravures ${ }^{36}$, sur le pastel de Boze (Musée Lambinet, Versailles) ou sur le buste de Deseine (Musée de la Révolution française, Vizille), le nez du député apparaît assez fin et légèrement retroussé. Sans même revenir sur les profondes marques de variole du masque, qu'aucun portrait ne peut laisser supposer aussi prononcées chez Robespierre, on peut s'étonner que cette différence physique n'ait pas suscité l'interrogation.

Si l'origine du masque n'est pas prouvée, si ni Marie Grosholtz, ni personne d'autre n'a pu fixer les traits de Robespierre après son exécution, que penser des analyses médicales rétrospectives du docteur Charlier ? Certes, il affirme également se reposer sur des sources. Il cite ainsi le témoignage de Pierre Villiers, mais sans prendre la peine d'en discuter l'authenticité, alors qu'il a été réfuté (à tort ou à raison) par René Garmy ${ }^{37}$. Les rares informations du docteur Souberbielle peuvent-elles alors suffire ? Et que dire d'une approche bibliographique qui met en exergue La vie privée de Robespierre de Nabonne, discutable en bien des points, et une surprenante interprétation d'un récent article de Peter McPhee dans les Annales historiques de la Révolution française, dont les analyses invitent au débat ${ }^{38}$ ? Non, aux yeux de l'historien, l'incertitude de l'information et l'absence de critique des sources invalident la démonstration et la prétendue

(34) Laura AURICCHIO, Adélaïde Labille-Guiard. Artist in the Age of Revolution, Los Angeles, The J. Paul Getty Museum, 2009, p. 77-79.

(35) Fernand BEAUCAMP, «Un portrait inconnu de Robespierre au musée de Lille », Revue du Nord, 1928, p. 21-34.

(36) Voir, par exemple, la gravure de Fiesinger, réalisée sous la Constituante à partir d'un dessin de Guérin.

(37) René GARMY, «Aux origines de la légende anti-robespierriste : Pierre Villiers et Robespierre », Actes du colloque Robespierre, Paris, SER, 1967, p. 19-33.

(38) http://www.theguardian.com/higher-education-network/blog/2014/feb/11/researchcitations-accuracy-media-responsibility?CMP=new_1194. 
découverte d'une sarcoïdose. Pour d' autres raisons, propres à leur discipline, plusieurs médecins ont également contesté le diagnostic ${ }^{39}$.

Dans l'attente de nouvelles révélations, promises pour les mois qui viennent, que retenir de cette affaire ? Elle montre tout d'abord, une nouvelle fois, combien les mécanismes de fabrication de l'histoire se sont à la fois accélérés, démocratisés et mondialisés. Matrice d'un nouveau folklore, internet a visiblement démultiplié les possibilités d'actualisation, de transformation et de diffusion des imaginaires collectifs souvent issus du passé le plus lointain : la rapidité et la liberté avec lesquelles ce nouvel avatar de Robespierre a été commenté, transformé, détourné et approprié par les internautes montre à quel point le passé se fabrique désormais également ailleurs que dans les livres, à la radio ou à la télévision, faisant des humanités digitales des territoires d'avenir pour ceux qui s'intéressent aux usages du passé. Cependant, cette énième affaire Robespierre a aussi prouvé que les historiens peuvent se saisir de ces outils pour exercer leur droit de vigilance, partager leur savoir en dehors de la sphère académique dans le temps court de l'actualité, sans pour autant renoncer aux règles de la méthode critique. La croyance collective dont a spontanément bénéficié le visage numérique de Robespierre démontre, quant à elle, la force des utopies «technologiques » prétendant décoder la véritable nature des individus à partir de leur apparence physique, depuis la physiognomonie des Lumières jusqu'à la biométrie actuelle en passant par la phrénologie et l'anthropologie criminelle du XIX ${ }^{\mathrm{e}}$ siècle. Faussement nouvelle, la démarche intellectuelle des auteurs du visage en 3D s'inscrit dans une longue histoire de la caractériologie humaine, revêtue des apparences de la modernité numérique. Cet épisode constitue donc un cas intéressant d'épistémologie de l'histoire : il montre combien la vérité historique ne se construit qu'en fonction de sources non seulement authentifiées, mais aussi choisies en fonction de questions précises et renouvelées. Pour les historiens de la Révolution française, l'affaire du « vrai visage de Robespierre » révèle, enfin, et une fois de plus, combien cette période et ses figures contribuent 
à dessiner, plus de deux siècles après, quelques profondes lignes de faille de la société française et de l'imaginaire occidental.

$$
\begin{array}{r}
\text { Hervé LEUWERS } \\
\text { Université Lille 3 - UMR-CNRS IRHiS } \\
\text { herve.leuwers @ univ-lille3.fr }
\end{array}
$$

Guillaume MAZEAU

Université Paris 1-Panthéon-Sorbonne - IHMC/IHRF mazeau.guillaume@free.fr 